
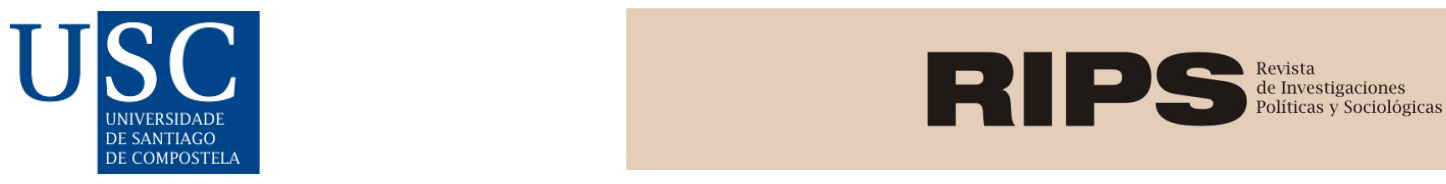

RIPS: Revista de Investigaciones Políticas y Sociológicas, 20(1), 2021. ISSN: 2255-5986

https://doi.org/10.15304/rips.20.1.7128

Artículos

\title{
Opinión pública europea y proceso de integración: el reto de la esfera pública supranacional
}

European public opinion and the integration process: the challenge of a supranational public sphere

\author{
José Miguel Rojo Martínez ${ }^{1}$, Alejandro Soler Contreras ${ }^{2}$ \\ ${ }^{1}$ Universidad de Murcia \\ ${ }^{2}$ Universidad de Murcia
}

Recibido: 24/09/2020; Aceptado: 03/02/2021

\section{Resumen}

Los académicos suelen discutir acerca de la inexistencia de la "esfera pública europea", si bien priman a este respecto las investigaciones desde una óptica teórica o conceptual. En un intento de cubrir esta laguna, el presente artículo contribuye al debate desde un enfoque empírico y cuantitativo, empleando datos del Eurobarómetro para comprobar el estado y las posibilidades de dicha esfera pública, encontrando que, aunque los ciudadanos europeos continúan prestando poca atención a las temáticas políticas europeas, existe cierto acuerdo en torno a los temas de la agenda pública.

Palabras clave: Opinión pública; Unión Europea; esfera pública; integración; demoscopia

\begin{abstract}
Scholars usually discuss the inexistence of a "European public sphere", but researches on this topic are mainly conceptual or theoretical reflections. Regarding the lack of empirical analysis on this issue, this paper aims to contribute to this debate from an empirical and quantitative approach, using Eurobarometer data to check the state and chances of this public sphere, finding out that European citizens still disregard European political debate, although there is a certain understanding on the public agenda.
\end{abstract}

Keywords: Public opinion; European Union; public sphere; integration; demoscopy

\section{Introducción}

Esta investigación se plantea una pregunta crucial para el proyecto europeo y para la investigación sobre comunicación en Europa: ¿existe una opinión pública europea? Si se prefiere, el término "opinión pública" puede ser sustituido, siguiendo a Habermas (1989), por el concepto de "esfera pública", lugar en el que se produce el advenimiento de la opinión pública, pero, en cualquier caso, de lo que se trata es de averiguar si podemos observar un espacio colectivo de debate, conversación y pensamiento supranacional o si, por el contrario, la opinión pública se

Copyright (c) Universidade de Santiago de Compostela. This is an open access article distributed under the terms of the Creative Commons Attribution-NonComercial-NoDerivatives 4.0 International (CC BY-NC-ND 4.0) License. 
mantiene como un hecho exclusivamente nacional, no logrando conformarse en un nivel europeo. Esto último supone un grave problema para el deseado proceso de integración (Bølstad, 2015; Nitoiu, 2012; Adam, 2015) que necesita de una ciudadanía capaz de identificarse con la comunidad política llamada Europa.

Koopmans (2007: 183-186) advierte cómo, al mismo tiempo, “las decisiones políticas en Europa se adoptan cada vez más en los ámbitos supranacional e intergubernamental”, pero, sin embargo, "el Estado-nación ha seguido siendo el centro principal de las identidades colectivas y la participación ciudadana". La clave para Koopmans no sería prestar atención a "la opinión pública medida en encuestas, es decir, en el nivel individual", sino que lo relevante es atender al "grado de europeización de los debates públicos y de la movilización política colectiva" y cómo este proceso se va visibilizando en "los medios europeos impresos", articuladores de la opinión pública.

La controversia sobre la existencia de una opinión pública europea aparece tímidamente en la literatura especializada española a través de investigaciones como la de Aguilera de Prat (2004: 168-176) en la que directamente se afirma que "al no existir una opinión pública europea, los ciudadanos siguen encapsulados en los Estados nacionales, de ahí que no razonen en términos europeos". Sin embargo, el mismo autor parece vislumbrar que "Europa podría existir como comunidad social y hay ciertos síntomas que parecen prefigurar la conformación de una opinión pública europea que los comparte”. Fernández Ostos (2013: 32-34) editó una interesante conversación entre los profesores Juan Torres y Sami Naïr precisamente sobre la condición de posibilidad de una esfera pública europea, llegando a la conclusión de que "la ausencia de un nexo común entre los europeos, más allá de la economía y de la moneda común, torna la esfera pública europea en una realidad imposible". El problema de la opinión pública europea es, en esencia, el problema del demos europeo, y así se expresa en las conclusiones de esta conversación: "no existe el pueblo europeo y por lo tanto no puede existir una esfera pública. Europa es un conjunto absorbente de 27 pueblos diferentes sin identidad común más allá de la económica".

Incluso siendo la posición anterior dominante, algunos autores, a propósito de la crisis económica de 2008 y sus consecuencias sobre el euro, han advertido "una cierta europeización de las esferas públicas (...), fenómeno en el que las cuestiones europeas se debaten cada vez más en los medios de comunicación nacionales". Aun considerando esta posibilidad, la débil formación de una opinión pública europea no ha sido superada y en ella intervienen, al menos, dos grandes factores. Por un lado, el relacionado con el sistema de medios. Por otro, el que se refiere a la arquitectura institucional (Ojala, 2013: 78).

Opinión pública y comunicación forman un binomio inseparable, por eso el estudio de la esfera pública lleva necesariamente aparejada una especial atención al ecosistema mediático que, realmente, materializa la opinión pública. Es recurrente, entonces, que los estudios sobre la opinión pública europea vengan siendo análisis de contenido sobre medios (Trenz, 2004) o reflexiones sobre el impacto de ciertos medios en la construcción y existencia de una esfera pública europea, como es el caso de Gripsrud (2007) sobre la televisión. Algunos de estos análisis incorporan la ausencia de medios de comunicación de masas europeos como desencadenante de los problemas de conformación del espacio público europeo, pero este factor no debe ser considerado aisladamente respecto de la influencia del particular sistema institucional, político y representativo de la Unión Europea, muchas veces calificado como deficitario a nivel democrático (Barón Goiriena, 2009) e incapaz de arbitrar un verdadero proceso de debate más allá de la interacción intergubernamental.

La falta de un nivel de deliberación pública puede considerarse un indicador más de los extensos problemas de legitimidad democrática de la Unión (Arregui, 2012; Nitoiu, 2012). Sobre el asunto 
particular de los medios europeos, Koopmans (2007: 185) recuerda que "ha habido algunos intentos de establecer medios de comunicación de masas a escala europea, pero la mayoría de ellos han desaparecido rápidamente (como el diario The European) o llevan una existencia marginal (por ejemplo, la cadena Euronews)". La falta de grandes medios a nivel europeo, contrasta con el éxito que sí han descrito "los medios transnacionales, capaces de crear un nicho en el panorama de los medios de comunicación", puntualiza Koopmans.

La aludida particularidad del sistema político-institucional de la Unión se añade al conjunto de factores que dificultan la formación de la pretendida esfera pública habida cuenta de la tendencia a la despolitización que alimenta. Este sesgo no hace más que alejar el debate político del ciudadano, que permanece ajeno o apático al devenir de la política europea, al favorecer el sistema institucional de la Unión mecanismos de funcionamiento que tienden a evitar la confrontación ideológica o la discusión abierta. Encontramos así un Europarlamento menos centrado en el juego de mayoría de gobierno/minoría de oposición (Rodríguez-Aguilera, 2015: 90) y el antagonismo ideológico, y más ligado al control del ejecutivo y la separación de poderes (Innerarity, 2017: 184-187), o una Comisión Europea cuyo Presidente elige el Europarlamento, pero cuyo método de elección de comisarios (uno por cada Gobierno de cada Estado Miembro, sea del color político que este sea) dificulta la existencia de un mandato político claro (Simón, 2018: 52), recibiendo además esta institución un tratamiento de "independiente" en un intento de "defender la policy de la politics" (Innerarity, 2017: 191).

Desde el diálogo teórico anterior podemos arribar hasta un interesante punto medio de consenso, que es el aportado por Eriksen (2005: 341): "la cooperación europea y la resolución de problemas crean espacios públicos, pero no han producido (hasta ahora) una esfera pública europea única y general. Más bien se trata de públicos transnacionales segmentados". Las conclusiones de Eriksen son reforzadas por los hallazgos de Baisnée (2007). El autor reflexiona sobre la equivocada concepción mayoritaria que trata a la esfera pública europea como una suerte de opinión pública nacional trasladada, cuando, en realidad, es una formación sectorial, selectiva y minoritaria, que no incluye a toda la ciudadanía, y que se limita a personas altamente implicadas, profesional o políticamente, con los problemas de la Unión. La condición no-mainstream de los debates europeos impide la calificación de la deliberación europea en términos de verdadera esfera pública. Y, de nuevo, el poco interés de los ciudadanos en los temas europeos o su escasa permeabilidad en el debate cotidiano no puede entenderse más que a partir de un constructo multifactorial necesariamente atravesado por la variable "identidad colectiva".

Si Habermas detectaba en 1994 que Europa sólo llegaría a "una política común si todos los países hablan sobre los mismos temas dándoles la misma relevancia” ${ }^{1}$, el no haber alcanzado ese objetivo décadas después no puede entenderse sin atender a la brecha cultural e identitaria, étnica y lingüística, y, también desde esta idea, podemos lograr nuevas formas de entendimiento de la opinión pública.

Dentro de las perspectivas teóricas dominantes en la explicación del complejo fenómeno de la opinión pública destaca la psicológica, aquella que concibe el concepto como "la suma de opiniones y actitudes individuales". La idea de que la opinión pública es un simple agregado de posiciones particulares nos impide con frecuencia explicar comportamientos de origen grupal. Frente a la teoría psicológica, el paradigma cultural "entiende la opinión pública como el conjunto de pensamientos y sentimientos colectivos, costumbres o tradiciones", por tanto, es un término "ligado a la fuerza de las creencias dominantes en una colectividad" (Moreno; Mora, 2016: 23-25). Si nos 
posicionamos en los postulados de la teoría psicológica la pregunta “existe una opinión pública europea?" es de fácil respuesta: sí. Es tan sencillo como entender que la misma es el sumatorio de las posiciones individuales de cada uno de los ciudadanos que viven en alguno de los países de la Unión, por heterogéneas que sean. Además, la puesta en marcha de instrumentos de medición como el Eurobarómetro, la encuesta referencial europea, o el European Values Study (EVS) sería una evidencia más de que, en efecto, hay un espacio público europeo con existencia constatable e independiente.

Ahora bien, muchos fenómenos parecen apuntar en otra dirección, como si cada país hablara consigo mismo o con otros países, pero no existiera un debate europeo más allá de los debates nacionales. Por ejemplo, las elecciones al Parlamento Europeo terminan librándose en todos los países miembros en clave nacional. Algunos estudios apuntan esta realidad, entre ellos el de Zugasti y Lafuente (2011: 142) que ven cómo "los comicios europeos no suscitaron interés por sí mismos, sino que, por el contrario, fueron abordados por los periódicos en clave nacional". La tradicional calificación de las Elecciones al Parlamento Europeo como comicios de segundo orden (Marsh, 1998) evidencia una jerarquía de subordinación de los temas europeos frente a las cuestiones más puramente domésticas que son las que terminan centrando los mensajes de los partidos en este tipo de competición electoral, usada muchas veces para fines de política interna. No obstante, el nacimiento en los últimos años de partidos euroescépticos y el impacto de algunos retos como el migratorio o el económico en el día a día de los Estados-nación ha provocado un destacado nivel de europeización de los debates nacionales (Seddone; Bobba; Roncarolo: 2019), que no debe ser confundido con el objeto de estudio estricto de este artículo. Que la opinión pública italiana, española o alemana hable más sobre Europa, no implica (necesariamente) que se esté constituyendo una deliberación coordinada y simultánea entre estas esferas nacionales.

Justo por lo anterior resulta necesario distinguir las dos grandes conceptualizaciones de la esfera pública europea. La primera atiende a "una esfera pública paneuropea que se extiende más allá de los Estados nacionales", se trata de concebir que "la esfera pública europea se asemeja a una esfera pública nacional, con una audiencia homogénea y un sistema de medios que se extiende hasta las mismas fronteras que la esfera pública". Los prerrequisitos de homogeneidad identitaria, mediática y cultural para Europa pueden considerarse excesivos y por demás innecesarios si atendemos a la realidad plural de algunos Estados. La superación teórica de la posición paneuropea sobre la esfera pública puede verse en un conjunto de trabajos que afirman percibir "desarrollarse una esfera pública europea a través de la europeización de la esfera pública nacional”. Digamos que esta segunda gran conceptualización no se preocupa tanto por la creación de una esfera pública supranacional típico-ideal, sino que entiende como evidencia de su existencia que "las esferas públicas nacionales se abran al debate sobre cuestiones con una dimensión europea, dando voz a los actores de las instituciones de la UE y de otros países miembros y a sus posiciones" (Adam, 2015: 372-373).

En la tensión entre el entendimiento clásico de una homogénea opinión pública y la moderna idea de la europeización, tratada ampliamente por autores como Buller y Gamble (2002), se sitúa esta investigación. La aportación más innovadora de este estudio es intentar responder a la recurrente pregunta con que se comenzaba este epígrafe a partir de datos de encuesta, complementando así los extensos y reiterados análisis teóricos y de medios. Se determinarán, a continuación, variables que son relevantes para concluir el nivel de conformación de la esfera pública europea, tanto en un sentido restrictivo como en una visión más flexible. Detectar indicadores viables que permitan la comparación y el análisis longitudinal por medio de series estadísticas debe entenderse como 
una metodología de investigación sobre opinión pública europea sencilla, pero, al mismo tiempo, efectiva en un momento todavía inicial de los trabajos de campo sobre el tema.

Si se negaba que la existencia de instrumentos de medición de actitudes individuales agregadas confirmara una esfera pública, en los datos siguientes se intentará comprobar con mayor claridad si esos mismos instrumentos demoscópicos nos informan con claridad de que los distintos países no hablan entre sí sobre lo mismo (independientemente de las posiciones) y que, incluso las teorías de la europeización de los debates nacionales son todavía empíricamente débiles, por entender con demasiada frecuencia que la presencia en la agenda mediática de un tema de política europea se entiende en el framing ciudadano como tal y no como un verdadero debate nacional más.

\section{Metodología}

Los datos empleados para realizar los análisis contenidos en este estudio provienen de diferentes preguntas seleccionadas del Eurobarómetro. Son, en consecuencia, datos secundarios de encuesta que toman como unidad de análisis a los ciudadanos de los diferentes países (no a los países en sí).

El periodo seleccionado es el 2018-2019 (considerando el promedio entre los dos estudios anuales que se realizan), buscando observar la secuencia longitudinal más reciente de evolución de las tendencias expresadas. Además, en mayo del año 2019, tuvo lugar la última elección del Parlamento Europeo, por lo que los resultados de este año adquieren una importancia reforzada. El método de investigación seleccionado es el estudio de caso, incorporando un enfoque estadístico y con un objetivo fundamentalmente descriptivo.

No procede plantear contrastación de hipótesis ya que la pregunta de investigación que sustenta el estudio no expresa una relación entre variables. En definitiva, se trata de, a partir de la descripción de datos de opinión pública, contribuir a la operacionalización del fenómeno, aportando al concepto una serie de dimensiones e indicadores que nos permitan medirlo en la realidad. Al operacionalizar queremos "obtener uno o varios indicadores empíricos de la manifestación de una propiedad o variable en un caso determinado" (Anduiza, Crespo y Méndez, 2009: 43). La cuidadosa elección de los indicadores abre la puerta a futuros trabajos que comprueben con mayor nivel de profundidad empírica, a través, por ejemplo, de análisis clasificatorios o multivariantes, posibles relaciones entre los elementos que integran la variable dependiente (esfera pública europea).

Así pues, examinando el objeto de nuestro estudio y las preguntas disponibles en nuestra fuente de datos, el Eurobarómetro estándar, tomamos como indicadores de la existencia de una esfera pública europea las preguntas QA4a, QA5 y D71a.1, aquellas que se refieren, respectivamente, a los dos mayores problemas percibidos por el encuestado a nivel personal y para la UE, y a la frecuencia de discusión sobre temas políticos nacionales, europeos o locales con el entorno del individuo (se adjuntan las tablas con los resultados).

La selección de estas preguntas se explica en tanto que, al tiempo que la D71a.1 nos permite comprobar si efectivamente se habla sobre temas europeos (clara precondición para la existencia de una esfera pública), las dos preguntas sobre problemáticas indagan en cuál sería el contenido de esta hipotética discusión, observando si, en la discusión sobre la política europea, se da un acuerdo en los temas a tratar y los issues que preocupan al ciudadano o si cada país se encuentra volcado hacia sus propios temas. Así, para poder realizar una suerte de medición de la esfera pública tomamos como componentes (dimensiones) de la misma, tal y como ilustra la tabla 1, la integración 
de la discusión sobre temas europeos en la vida cotidiana del ciudadano y el acuerdo en los temas de debate de la agenda pública.

\begin{tabular}{|c|c|c|}
\hline Concepto & Dimensiones & Indicadores \\
\hline \multirow{3}{*}{$\begin{array}{l}\text { Opinión } \\
\text { pública } \\
\text { europea }\end{array}$} & $\begin{array}{l}\text { Integración de la } \\
\text { discusión sobre } \\
\text { temas europeos } \\
\text { en la vida } \\
\text { cotidiana }\end{array}$ & $\begin{array}{c}\text { Frecuencia de } \\
\text { discusión de temas } \\
\text { políticos en el entorno } \\
\text { del encuestado } \\
\text { (D71a.1) }\end{array}$ \\
\hline & \multirow[t]{2}{*}{$\begin{array}{l}\text { Nivel de } \\
\text { acuerdo entre } \\
\text { los temas de } \\
\text { debate de la } \\
\text { agenda pública }\end{array}$} & $\begin{array}{c}\text { Problemas que } \\
\text { personalmente más } \\
\text { afectan al encuestado, } \\
\text { con referencia a su } \\
\text { contexto nacional } \\
\text { (QA4a) }\end{array}$ \\
\hline & & $\begin{array}{c}\text { Problemas principales } \\
\text { que el encuestado cree } \\
\text { que afronta la UE } \\
\text { (QA5) }\end{array}$ \\
\hline
\end{tabular}

Operacionalización del concepto "opinión pública europea”

Fuente: Elaboración propia

\section{Discusión de resultados}

El análisis de la pregunta D71.a.1 (Tabla 2), relativa a la frecuencia de discusión de temas políticos en el entorno del encuestado, revela un patrón claro que se mantiene sin variaciones sustanciales a lo largo del tiempo y para los distintos países europeos. En una mirada general, tal y como indica la tabla 2, los niveles de "discusión política" se estabilizan en cotas moderadas, predominando la opción "ocasionalmente" con un valor cercano a los 50 puntos. Eso sí, el interés político no es homogéneo entre las distintas esferas territoriales. Si bien la respuesta "ocasionalmente" predomina tanto en la arena local como en la nacional y la europea con similares puntuaciones, es el nivel europeo el que registra mayor cantidad de encuestados que declaran no discutir "nunca" con su entorno sobre temas de política europea. Haciendo la media de la serie histórica 2018-2019, esta opción representa un 23,25\% para la política nacional y un 27,25\% para la local, mientras a nivel europeo se encuentra en un 33,75\%. Igualmente, mientras que el porcentaje de encuestados que 
discute "frecuentemente" de cuestiones políticas nacionales y locales es de un 23,50\% y 20,75\% respectivamente, para la política europea el porcentaje es de un escaso 15,25\%.

Tabla 2. Resultados (\%) para la pregunta D71a.1 sobre frecuencia de discusión sobre temas políticos por nivel territorial 2018 y $2019(a+b)$ con promedio 3

\begin{tabular}{|l|l|l|l|}
\hline $\begin{array}{l}\text { Cuando te reúnes } \\
\text { con amigos o } \\
\text { familiares, ddirías } \\
\text { que hablas }\end{array}$ & $\begin{array}{l}\text { Temas de política } \\
\text { nacional }\end{array}$ & $\begin{array}{l}\text { Temas de política } \\
\text { europea }\end{array}$ & $\begin{array}{l}\text { Temas de política } \\
\text { local }\end{array}$ \\
\hline Año 2018 (UE 28) & Año 2018 (UE 28) & Año 2018 (UE 28) & Año 2018 (UE 28) \\
\hline Frecuentemente & $23,5 \%$ & $15,0 \%$ & $21,0 \%$ \\
\hline Ocasionalmente & $54,0 \%$ & $52,0 \%$ & $52,5 \%$ \\
\hline Nunca & $22,5 \%$ & $33,0 \%$ & $26,5 \%$ \\
\hline NS/NC & - & - & - \\
\hline Año 2019 (UE 28) & Año 2019 (UE 28) & Año 2019 (UE 28) & Año 2019 (UE 28) \\
\hline Frecuentemente & $23,5 \%$ & $15,5 \%$ & $20,5 \%$ \\
\hline Ocasionalmente & $52,5 \%$ & $50,0 \%$ & $51,5 \%$ \\
\hline Nunca & $24,0 \%$ & $34,5 \%$ & $28,0 \%$ \\
\hline NS/NC & - & - & - \\
\hline Promedio (UE 28) & Promedio (UE 28) & Promedio (UE 28) & Promedio (UE 28) \\
\hline Frecuentemente & $23,5 \%$ & $15,25 \%$ & $20,75 \%$ \\
\hline Ocasionalmente & $53,25 \%$ & $51,0 \%$ & $52,0 \%$ \\
\hline Nunca & $23,25 \%$ & $33,75 \%$ & $27,25 \%$ \\
\hline NS/NC & - & - & - \\
\hline & & & \\
\hline
\end{tabular}

Resultados (\%) para la pregunta D71a.1 sobre frecuencia de discusión sobre temas políticos por nivel territorial 2018 y $2019(a+b)$ con promedio 3

Fuente: Elaboración propia a partir de Eurobarómetro 2018 y 2019

Cierto es que partimos del contexto de sociedades democráticas desafectas donde, como podemos ver en la pregunta revisada, los niveles generales de discusión política son moderados o bajos, pero, dentro de este esquema, los datos para lo propiamente europeo son especialmente negativos. El hecho de que la política europea sea la que registra un menor interés o presencia en la vida del encuestado conecta con la conocida teoría de las "elecciones de segundo orden" de Reif y Schmitt (1980). Desde esta perspectiva, podemos asumir que, para el ciudadano, la política europea 
es una "política de segundo orden" a la que, en definitiva, no presta una gran atención y por la que no muestra excesivo interés. Las consecuencias para nuestra investigación son inmediatas: ¿cómo se va a desarrollar una esfera pública europea o una opinión pública si no se discute sobre temas europeos? La atención al espacio de discusión vincula la emergencia de un entorno de deliberación.

Este déficit en la discusión sobre temáticas europeas plantea un serio inconveniente al desarrollo de la esfera pública, puesto que puede implicar tanto una falta de interés latente como una falta de información. Es realmente difícil establecer la dirección de la relación causal entre estas dos variables, toda vez que la falta de información puede derivarse del conocido poco interés que los espectadores prestan a la información europea, pero también este bajo interés se deriva de las propias decisiones de los medios sobre primacía y enfoque de los contenidos. Conectando este punto con las cuestiones que discutíamos anteriormente, debemos señalar, de nuevo, la inexistencia de un sistema de medios propiamente europeo y el diseño institucional de la Unión como elementos que dificultan la aparición del necesario espacio de interacción comunicativa. Cabría una reflexión mayor: ¿es posible despertar interés sobre los temas europeos? Tal vez el propio espacio de competencias de la Unión hace imposible convertir sus debates en discusiones domésticas, por mucho que sepamos que el marco normativo y decisional de este nivel termina vinculando muchas de las acciones cotidianas, pero el impacto real necesita de una acción nacional o regional que es la que, por su carácter concreto y más simple, finalmente recibe la atención mediática.

Si transitamos de la existencia de la discusión política a qué podría ser su contenido ("de qué se habla"), las problemáticas que se discuten, la pregunta QA4a (los dos problemas que personalmente más afectan al encuestado) muestra un consenso limitado en torno a algunos temas que se repiten habitualmente como principales preocupaciones del conjunto de países de la Unión (inflación/ coste de vida, salud y seguridad social, pensiones, finanzas del hogar e impuestos). 
Tabla 3. Media de respuestas para 2018 (a+b) a la cuestión QA4a (los dos problemas que más preocupan al encuestado personalmente $)^{4}$

\begin{tabular}{|c|c|c|c|c|c|c|c|c|c|c|c|c|c|c|}
\hline $\begin{array}{c}\text { Problemas que más } \\
\text { preocupan } \\
\text { personalmente }\end{array}$ & $\begin{array}{l}\mathrm{UE} \\
28\end{array}$ & $\begin{array}{l}\text { D- } \\
\text { W }\end{array}$ & IE & EL & ES & IT & $\mathrm{CY}$ & LU & MT & NL & RO & SI & FI & SE \\
\hline $\begin{array}{c}\text { Situación económica } \\
\text { en mi país }\end{array}$ & $\begin{array}{l}8.0 \\
\%\end{array}$ & $\begin{array}{l}2,5 \\
\%\end{array}$ & $7,0 \%$ & $\begin{array}{c}14,5 \\
\%\end{array}$ & $\begin{array}{l}13, \\
5 \%\end{array}$ & $\begin{array}{l}10, \\
0 \%\end{array}$ & $\begin{array}{l}18, \\
5 \%\end{array}$ & $\begin{array}{r}2,5 \\
\%\end{array}$ & $\begin{array}{l}2,5 \\
\%\end{array}$ & $5,0 \%$ & $\begin{array}{l}13 \\
0 \%\end{array}$ & $\begin{array}{l}6,0 \\
\%\end{array}$ & $\begin{array}{l}11, \\
0 \%\end{array}$ & $\begin{array}{l}6,0 \\
\%\end{array}$ \\
\hline $\begin{array}{c}\text { Precios } \\
\text { crecientes } / \text { coste de la } \\
\text { vida }\end{array}$ & $\begin{array}{l}30, \\
5 \%\end{array}$ & $\begin{array}{c}19,5 \\
\%\end{array}$ & $\begin{array}{c}41,5 \\
\%\end{array}$ & $\begin{array}{c}27,5 \\
\%\end{array}$ & $\begin{array}{l}25, \\
0 \%\end{array}$ & $\begin{array}{l}23, \\
0 \%\end{array}$ & $\begin{array}{l}28, \\
0 \%\end{array}$ & $\begin{array}{l}32 \\
0 \%\end{array}$ & $\begin{array}{l}28 \\
5 \%\end{array}$ & $\begin{array}{c}19,0 \\
\%\end{array}$ & $\begin{array}{l}33 \\
0 \%\end{array}$ & $\begin{array}{l}17 \\
0 \%\end{array}$ & $\begin{array}{l}14 \\
0 \%\end{array}$ & $\begin{array}{l}7,0 \\
\%\end{array}$ \\
\hline Impuestos & $\begin{array}{l}12, \\
0 \%\end{array}$ & $\begin{array}{l}7,5 \\
\% \\
\end{array}$ & $\begin{array}{l}12,0 \\
\%\end{array}$ & $\begin{array}{c}28,0 \\
\%\end{array}$ & $\begin{array}{l}15, \\
5 \%\end{array}$ & $\begin{array}{l}24, \\
5 \% \\
\end{array}$ & $\begin{array}{r}4,5 \\
\% \\
\end{array}$ & $\begin{array}{r}9,0 \\
\% \\
\end{array}$ & $\begin{array}{r}4,5 \\
\%\end{array}$ & $6,0 \%$ & $\begin{array}{l}10 \\
0 \%\end{array}$ & $\begin{array}{l}9,5 \\
\% \\
\end{array}$ & $\begin{array}{l}7,0 \\
\% \\
\end{array}$ & $\begin{array}{r}5,5 \\
\% \\
\end{array}$ \\
\hline Desempleo & $\begin{array}{l}10, \\
0 \%\end{array}$ & $\begin{array}{c}4,5 \\
\%\end{array}$ & $\begin{array}{c}10,0 \\
\%\end{array}$ & $\begin{array}{c}18,0 \\
\%\end{array}$ & $\begin{array}{l}24, \\
5 \%\end{array}$ & $\begin{array}{l}18, \\
0 \%\end{array}$ & $\begin{array}{l}17 \\
0 \%\end{array}$ & $\begin{array}{l}6,5 \\
\%\end{array}$ & $\begin{array}{l}1,0 \\
\%\end{array}$ & $4,5 \%$ & $\begin{array}{l}6,0 \\
\%\end{array}$ & $\begin{array}{l}8,0 \\
\%\end{array}$ & $\begin{array}{l}6,5 \\
\%\end{array}$ & $\begin{array}{r}4,5 \\
\%\end{array}$ \\
\hline Vivienda & $\begin{array}{l}6,5 \\
\%\end{array}$ & $\begin{array}{c}9,5 \\
\%\end{array}$ & $\begin{array}{c}16,5 \\
\%\end{array}$ & $0,5 \%$ & $\begin{array}{r}5,5 \\
\%\end{array}$ & $\begin{array}{l}3,0 \\
\%\end{array}$ & $\begin{array}{l}6,5 \\
\%\end{array}$ & $\begin{array}{l}22, \\
0 \%\end{array}$ & $\begin{array}{l}8,0 \\
\%\end{array}$ & $7,5 \%$ & $\begin{array}{l}6,0 \\
\%\end{array}$ & $\begin{array}{l}5,5 \\
\%\end{array}$ & $\begin{array}{l}11 \\
5 \%\end{array}$ & $\begin{array}{c}10,5 \\
\%\end{array}$ \\
\hline $\begin{array}{c}\text { Situación financiera } \\
\text { de mi hogar }\end{array}$ & $\begin{array}{l}13 \\
5 \%\end{array}$ & $\begin{array}{l}6,5 \\
\%\end{array}$ & $\begin{array}{c}11,5 \\
\%\end{array}$ & $\begin{array}{c}29,5 \\
\%\end{array}$ & $\begin{array}{l}10 \\
0 \%\end{array}$ & $\begin{array}{l}10, \\
5 \%\end{array}$ & $\begin{array}{l}28, \\
5 \%\end{array}$ & $\begin{array}{l}8,0 \\
\%\end{array}$ & $\begin{array}{r}5,5 \\
\%\end{array}$ & $\begin{array}{c}13,5 \\
\%\end{array}$ & $\begin{array}{l}15 \\
0 \%\end{array}$ & $\begin{array}{l}16, \\
0 \%\end{array}$ & $\begin{array}{l}22, \\
5 \%\end{array}$ & $\begin{array}{c}12,0 \\
\%\end{array}$ \\
\hline $\begin{array}{c}\text { Salud y seguridad } \\
\text { social }\end{array}$ & $\begin{array}{l}17 \\
5 \%\end{array}$ & $\begin{array}{c}15,0 \\
\%\end{array}$ & $\begin{array}{c}24,5 \\
\%\end{array}$ & $\begin{array}{c}13,5 \\
\%\end{array}$ & $\begin{array}{l}11 \\
5 \%\end{array}$ & $\begin{array}{l}13, \\
0 \%\end{array}$ & $\begin{array}{l}16, \\
0 \%\end{array}$ & $\begin{array}{l}9,0 \\
\%\end{array}$ & $\begin{array}{l}10 \\
0 \%\end{array}$ & $\begin{array}{c}32,5 \\
\%\end{array}$ & $\begin{array}{l}22 \\
0 \%\end{array}$ & $\begin{array}{l}17 \\
5 \%\end{array}$ & $\begin{array}{l}41 \\
5 \%\end{array}$ & $\begin{array}{l}45,5 \\
\%\end{array}$ \\
\hline Sistema educativo & $\begin{array}{l}10, \\
0 \% \\
\end{array}$ & $\begin{array}{c}14,0 \\
\%\end{array}$ & $9,5 \%$ & $9,0 \%$ & $\begin{array}{l}9,0 \\
\%\end{array}$ & $\begin{array}{l}7,0 \\
\% \\
\end{array}$ & $\begin{array}{l}11, \\
5 \% \\
\end{array}$ & $\begin{array}{l}17 \\
5 \% \\
\end{array}$ & $\begin{array}{l}6,0 \\
\% \\
\end{array}$ & $\begin{array}{c}22,5 \\
\%\end{array}$ & $\begin{array}{l}8,0 \\
\% \\
\end{array}$ & $\begin{array}{l}8,0 \\
\% \\
\end{array}$ & $\begin{array}{l}15, \\
0 \% \\
\end{array}$ & $\begin{array}{c}18,5 \\
\%\end{array}$ \\
\hline $\begin{array}{l}\text { Medioambiente, } \\
\text { clima y energia }\end{array}$ & $\begin{array}{l}9,5 \\
\%\end{array}$ & $\begin{array}{c}14,0 \\
\%\end{array}$ & $9,5 \%$ & $5,0 \%$ & $\begin{array}{l}5,0 \\
\%\end{array}$ & $\begin{array}{l}6,0 \\
\%\end{array}$ & $\begin{array}{l}3,5 \\
\%\end{array}$ & $\begin{array}{l}13, \\
0 \%\end{array}$ & $\begin{array}{l}22 \\
0 \%\end{array}$ & $\begin{array}{c}27,0 \\
\%\end{array}$ & $\begin{array}{l}5,0 \\
\%\end{array}$ & $\begin{array}{l}5,0 \\
\%\end{array}$ & $\begin{array}{l}28, \\
5 \%\end{array}$ & $\begin{array}{c}30,5 \\
\%\end{array}$ \\
\hline Pensiones & $\begin{array}{l}16, \\
0 \%\end{array}$ & $\begin{array}{c}15,0 \\
\%\end{array}$ & $9,0 \%$ & $\begin{array}{c}22,0 \\
\%\end{array}$ & $\begin{array}{l}22 \\
0 \%\end{array}$ & $\begin{array}{l}15, \\
0 \%\end{array}$ & $\begin{array}{l}9,0 \\
\%\end{array}$ & $\begin{array}{l}6,0 \\
\%\end{array}$ & $\begin{array}{l}14 \\
0 \%\end{array}$ & $\begin{array}{c}16,5 \\
\%\end{array}$ & $\begin{array}{l}16 \\
0 \%\end{array}$ & $\begin{array}{l}19, \\
0 \%\end{array}$ & $\begin{array}{l}15, \\
5 \%\end{array}$ & $\begin{array}{c}13,5 \\
\%\end{array}$ \\
\hline Condiciones de vida & $\begin{array}{l}8,5 \\
\%\end{array}$ & $\begin{array}{l}6,0 \\
\%\end{array}$ & $4,5 \%$ & $7,5 \%$ & $\begin{array}{l}7,5 \\
\%\end{array}$ & $\begin{array}{l}11, \\
0 \%\end{array}$ & $\begin{array}{l}5,5 \\
\%\end{array}$ & $\begin{array}{l}4,5 \\
\%\end{array}$ & $\begin{array}{l}5,5 \\
\%\end{array}$ & $6,5 \%$ & $\begin{array}{l}18 \\
5 \%\end{array}$ & $\begin{array}{l}15 \\
5 \%\end{array}$ & $\begin{array}{l}16 \\
5 \%\end{array}$ & $\begin{array}{c}9,0 \\
\%\end{array}$ \\
\hline
\end{tabular}

Media de respuestas para $2018(\mathrm{a}+\mathrm{b})$ a la cuestión QA4a (los dos problemas que más preocupan al encuestado personalmente $)^{4}$

Fuente: Elaboración propia a partir de Eurobarómetro 2018 
Tabla 4. Media de respuestas para 2019 (a+b) a la cuestión QA4a (los dos problemas que más preocupan al encuestado personalmente $)^{5}$

\begin{tabular}{|c|c|c|c|c|c|c|c|c|c|c|c|c|c|c|}
\hline $\begin{array}{c}\text { Problemas que más } \\
\text { preocupan } \\
\text { personalmente }\end{array}$ & UE28 & EL & ES & IT & $\mathrm{CY}$ & LV & LT & $\begin{array}{l}\mathrm{L} \\
\mathrm{U}\end{array}$ & $\mathrm{NL}$ & PL & $\mathrm{PT}$ & RO & SI & SE \\
\hline Crimen & $6.0 \%$ & $\begin{array}{c}8,0 \\
\%\end{array}$ & $\begin{array}{c}3, \\
0 \\
0\end{array}$ & $\begin{array}{l}6,0 \\
\%\end{array}$ & $\begin{array}{l}13, \\
0 \%\end{array}$ & $\begin{array}{l}1,5 \\
\%\end{array}$ & $\begin{array}{c}1,0 \\
\%\end{array}$ & $\begin{array}{l}6 \\
5\end{array}$ & $\begin{array}{l}4,0 \\
\%\end{array}$ & $\begin{array}{c}4,0 \\
\%\end{array}$ & $\begin{array}{l}0,5 \\
\%\end{array}$ & $\begin{array}{c}6,5 \\
\%\end{array}$ & $\begin{array}{l}1,0 \\
\%\end{array}$ & $\begin{array}{c}14,5 \\
\%\end{array}$ \\
\hline $\begin{array}{l}\text { Precios crecientes } \\
\text { /Coste de la vida }\end{array}$ & $31,0 \%$ & $\begin{array}{l}28, \\
0 \%\end{array}$ & $\begin{array}{r}23 \\
, 0 \\
0\end{array}$ & $\begin{array}{c}24,5 \\
\%\end{array}$ & $\begin{array}{l}43, \\
0 \%\end{array}$ & $\begin{array}{l}39 \\
0 \%\end{array}$ & $\begin{array}{l}61 \\
0 \%\end{array}$ & $\begin{array}{r}28 \\
, 0 \\
0\end{array}$ & $\begin{array}{l}19, \\
5 \%\end{array}$ & $\begin{array}{l}43 \\
5 \%\end{array}$ & $\begin{array}{l}39 \\
5 \%\end{array}$ & $\begin{array}{l}33 \\
0 \%\end{array}$ & $\begin{array}{l}16 \\
5 \%\end{array}$ & $\begin{array}{l}5,5 \\
\%\end{array}$ \\
\hline Impuestos & $10,5 \%$ & $\begin{array}{l}27, \\
0 \%\end{array}$ & $\begin{array}{l}14 \\
, 0\end{array}$ & $\begin{array}{c}23,0 \\
\%\end{array}$ & $\begin{array}{l}10 \\
0 \%\end{array}$ & $\begin{array}{l}21 \\
0 \%\end{array}$ & $\begin{array}{l}20, \\
0 \%\end{array}$ & $\begin{array}{c}9 \\
5\end{array}$ & $\begin{array}{l}3,5 \\
\%\end{array}$ & $\begin{array}{l}7,0 \\
\%\end{array}$ & $\begin{array}{l}20 \\
0 \%\end{array}$ & $\begin{array}{l}10 \\
5 \%\end{array}$ & $\begin{array}{l}7,0 \\
\%\end{array}$ & $\begin{array}{r}4,0 \\
\%\end{array}$ \\
\hline Desempleo & $9,5 \%$ & $\begin{array}{l}19 \\
0 \%\end{array}$ & $\begin{array}{r}19 \\
, 5\end{array}$ & $\begin{array}{c}18,5 \\
\%\end{array}$ & $\begin{array}{l}29, \\
5 \%\end{array}$ & $\begin{array}{l}7,5 \\
\%\end{array}$ & $\begin{array}{c}7,0 \\
\%\end{array}$ & $\begin{array}{c}4 \\
5\end{array}$ & $\begin{array}{l}4,0 \\
\%\end{array}$ & $\begin{array}{c}5,0 \\
\%\end{array}$ & $\begin{array}{l}8,0 \\
\%\end{array}$ & $\begin{array}{c}6,0 \\
\%\end{array}$ & $\begin{array}{l}6,5 \\
\%\end{array}$ & $\begin{array}{r}4,0 \\
\%\end{array}$ \\
\hline Vivienda & $8,0 \%$ & $\begin{array}{c}0,5 \\
\%\end{array}$ & $\begin{array}{l}10 \\
, 0\end{array}$ & $\begin{array}{c}4,0 \\
\%\end{array}$ & $\begin{array}{l}18 \\
5 \%\end{array}$ & $\begin{array}{l}8,0 \\
\%\end{array}$ & $\begin{array}{c}5,5 \\
\%\end{array}$ & $\begin{array}{r}24 \\
5\end{array}$ & $\begin{array}{l}7,5 \\
\%\end{array}$ & $\begin{array}{c}5,5 \\
\%\end{array}$ & $\begin{array}{l}5,5 \\
\%\end{array}$ & $\begin{array}{c}6,5 \\
\%\end{array}$ & $\begin{array}{l}7,5 \\
\%\end{array}$ & $\begin{array}{l}9,5 \\
\%\end{array}$ \\
\hline $\begin{array}{l}\text { Situación financiera } \\
\text { de mi hogar }\end{array}$ & $12,5 \%$ & $\begin{array}{l}25, \\
0 \%\end{array}$ & $\begin{array}{c}8, \\
5 \\
0\end{array}$ & $\begin{array}{c}11,0 \\
\%\end{array}$ & $\begin{array}{l}40, \\
5 \%\end{array}$ & $\begin{array}{l}9,0 \\
\%\end{array}$ & $\begin{array}{l}11 \\
5 \%\end{array}$ & $\begin{array}{c}7 \\
5 \\
0\end{array}$ & $\begin{array}{r}9,5 \\
\%\end{array}$ & $\begin{array}{l}13, \\
0 \%\end{array}$ & $\begin{array}{l}9,0 \\
\%\end{array}$ & $\begin{array}{l}15 \\
0 \%\end{array}$ & $\begin{array}{l}12, \\
5 \%\end{array}$ & $\begin{array}{c}13,0 \\
\%\end{array}$ \\
\hline Inmigración & $5,0 \%$ & $\begin{array}{c}6,0 \\
\% \\
\end{array}$ & $\begin{array}{c}3 \\
5\end{array}$ & $\begin{array}{l}8,5 \\
\% \\
\end{array}$ & $\begin{array}{l}24, \\
5 \%\end{array}$ & $\begin{array}{l}1,0 \\
\%\end{array}$ & $\begin{array}{l}1,5 \\
\% \\
\end{array}$ & $\begin{array}{c}4, \\
0\end{array}$ & $\begin{array}{r}3,5 \\
\% \\
\end{array}$ & $\begin{array}{c}2,5 \\
\%\end{array}$ & $\begin{array}{l}2,0 \\
\%\end{array}$ & $\begin{array}{r}4,5 \\
\% \\
\end{array}$ & $\begin{array}{l}4,0 \\
\%\end{array}$ & $\begin{array}{l}9,5 \\
\%\end{array}$ \\
\hline $\begin{array}{c}\text { Salud y seguridad } \\
\text { social }\end{array}$ & $18,5 \%$ & $\begin{array}{l}14, \\
0 \%\end{array}$ & $\begin{array}{l}12 \\
, 5 \\
0\end{array}$ & $\begin{array}{c}11,5 \\
\%\end{array}$ & $\begin{array}{l}24, \\
0 \%\end{array}$ & $\begin{array}{l}30 \\
5 \%\end{array}$ & $\begin{array}{l}17 \\
0 \%\end{array}$ & $\begin{array}{l}6, \\
5\end{array}$ & $\begin{array}{l}33, \\
0 \%\end{array}$ & $\begin{array}{l}19, \\
0 \%\end{array}$ & $\begin{array}{l}25 \\
0 \%\end{array}$ & $\begin{array}{l}23 \\
5 \%\end{array}$ & $\begin{array}{l}20 \\
0 \%\end{array}$ & $\begin{array}{c}43,0 \\
\%\end{array}$ \\
\hline Sistema educativo & $10,0 \%$ & $\begin{array}{c}9,0 \\
\%\end{array}$ & $\begin{array}{r}10 \\
, 5 \\
0\end{array}$ & $\begin{array}{l}7,5 \\
\%\end{array}$ & $\begin{array}{l}14, \\
5 \%\end{array}$ & $\begin{array}{l}10 \\
0 \%\end{array}$ & $\begin{array}{l}7,5 \\
\%\end{array}$ & $\begin{array}{r}13 \\
, 5 \\
0\end{array}$ & $\begin{array}{l}23, \\
0 \%\end{array}$ & $\begin{array}{l}5,5 \\
\%\end{array}$ & $\begin{array}{l}9,0 \\
\%\end{array}$ & $\begin{array}{c}9,5 \\
\%\end{array}$ & $\begin{array}{l}7,5 \\
\%\end{array}$ & $\begin{array}{c}18,0 \\
\%\end{array}$ \\
\hline $\begin{array}{l}\text { Medioambiente, } \\
\text { clima y energía }\end{array}$ & $14,5 \%$ & $\begin{array}{l}1,5 \\
\%\end{array}$ & $\begin{array}{l}8, \\
0 \\
\%\end{array}$ & $\begin{array}{l}8,0 \\
\%\end{array}$ & $\begin{array}{l}8,0 \\
\%\end{array}$ & $\begin{array}{c}4,0 \\
\%\end{array}$ & $\begin{array}{c}4,0 \\
\%\end{array}$ & $\begin{array}{l}20 \\
, 0 \\
\%\end{array}$ & $\begin{array}{l}37, \\
5 \%\end{array}$ & $\begin{array}{c}8,5 \\
\%\end{array}$ & $\begin{array}{l}4,0 \\
\%\end{array}$ & $\begin{array}{c}8,5 \\
\%\end{array}$ & $\begin{array}{l}7,0 \\
\%\end{array}$ & $\begin{array}{c}35,0 \\
\%\end{array}$ \\
\hline Pensiones & $15,0 \%$ & $\begin{array}{l}17 \\
5 \%\end{array}$ & $\begin{array}{r}21 \\
, 0\end{array}$ & $\begin{array}{c}13,5 \\
\%\end{array}$ & $\begin{array}{r}9,5 \\
\%\end{array}$ & $\begin{array}{l}18 \\
0 \%\end{array}$ & $\begin{array}{l}16, \\
0 \%\end{array}$ & $\begin{array}{l}3 \\
5\end{array}$ & $\begin{array}{l}23, \\
0 \%\end{array}$ & $\begin{array}{l}12 \\
5 \%\end{array}$ & $\begin{array}{l}19 \\
5 \%\end{array}$ & $\begin{array}{l}15 \\
0 \%\end{array}$ & $\begin{array}{l}19 \\
5 \%\end{array}$ & $\begin{array}{c}13,5 \\
\%\end{array}$ \\
\hline Condiciones de vida & $8,5 \%$ & $\begin{array}{l}10, \\
0 \%\end{array}$ & $\begin{array}{c}8, \\
0 \\
\%\end{array}$ & $\begin{array}{c}10,0 \\
\%\end{array}$ & $\begin{array}{l}7,0 \\
\%\end{array}$ & $\begin{array}{l}5,0 \\
\%\end{array}$ & $\begin{array}{c}5,0 \\
\%\end{array}$ & $\begin{array}{c}4, \\
5 \\
\%\end{array}$ & $\begin{array}{r}4,5 \\
\%\end{array}$ & $\begin{array}{l}15 \\
5 \%\end{array}$ & $\begin{array}{l}8,5 \\
\%\end{array}$ & $\begin{array}{l}16, \\
5 \%\end{array}$ & $\begin{array}{l}16, \\
5 \%\end{array}$ & $\begin{array}{l}8,0 \\
\%\end{array}$ \\
\hline
\end{tabular}

Media de respuestas para $2019(\mathrm{a}+\mathrm{b})$ a la cuestión QA4a (los dos problemas que más preocupan al encuestado personalmente $)^{5}$

Fuente: Elaboración propia a partir de Eurobarómetro 2019

Aunque puede haber ciertas diferencias en el grado de preocupación, estos issues tienden a ocupar los primeros puestos en todos los países. Al margen de esto, varios países cuentan con problemáticas diferenciales, que los hacen divergir puntualmente del resto de la Unión. Así, si bien el paro ha sido en ocasiones preocupación general entre los países europeos, su problemática se encuentra especialmente instalada, de acuerdo con las tablas 3 y 4, en países del Sur como España, Grecia, Italia o Chipre (respectivamente, 24,5\%, 27\%, 18,5\% y 29,5\% de porcentajes máximos a 
lo largo de la serie). Continuando en el Sur europeo, además del paro, Chipre y Grecia comparten una preocupación frecuente sobre la situación económica general del país. Mientras en el conjunto de la Unión este problema suele obtener una puntuación de 8\%, en ambos países se sitúa en un $18,5 \%$ y 14,0\% en 2018. España, sin llegar a los niveles griegos o chipriotas, se aproxima a ellos en resultados para esta cuestión.

Suecia, Holanda y Finlandia comparten una preocupación continua por el sistema educativo que no se refleja en otros países, llegando a un elevado 23\% en Holanda en 2019. Los mismos países, junto con Malta, repiten en la cuestión del cambio climático como asunto destacado (si bien este tema comienza a popularizarse en la Unión en el 2019). Especialmente llamativo es el caso sueco, donde, en 2018, la preocupación por el cambio climático llega al 30,5\%.

Por último, con un perfil de países menos marcado, la preocupación por la inmigración despunta de forma intermitente en regiones como Chipre, Suecia, Italia o Malta. Por ejemplo, en 2019 era de un $24,5 \%$ para Chipre y de un $5 \%$ para el conjunto de la UE.

Atendiendo ahora a la pregunta QA5a, los dos problemas principales que el encuestado cree que afronta la UE, lo primero que observamos es que las respuestas no guardan relación con la percepción de los encuestados sobre los dos principales problemas personales que afrontan en sus vidas. Repitiendo el anterior sistema de análisis, los problemas percibidos para la Unión que mayores resultados obtienen para el conjunto de países a lo largo de la serie temporal son la inmigración, el terrorismo, la situación económica, las finanzas de los Estados Miembros y, en menor medida, el desempleo y el cambio climático. En un cierto paralelismo con la pregunta de las principales preocupaciones a nivel personal del encuestado, observamos que, si bien desempleo y cambio climático cuentan entre los principales problemas para el conjunto de países, el primero preocupa especialmente a países como Italia, Chipre, España y Grecia, mientras el segundo capta una elevada atención de Suecia, Finlandia u Holanda. 
Tabla 5. Media de respuestas para el año $2018(\mathrm{a}+\mathrm{b})$ a la cuestión QA5 (los dos problemas que el encuestado considera principales para la UE) ${ }^{6}$

\begin{tabular}{c|c|c}
\hline $\begin{array}{c}\text { Dos principales } \\
\text { problemas de la UE }\end{array}$ & UE 28 & UE 28-UK \\
\hline $\begin{array}{c}\text { La situación } \\
\text { económica en mi país }\end{array}$ & $18,0 \%$ & $17,0 \%$ \\
\hline El terrorismo & $16,5 \%$ & $17,0 \%$ \\
\hline $\begin{array}{c}\text { La situación } \\
\text { financiera de mi } \\
\text { hogar }\end{array}$ & $16,5 \%$ & $17,0 \%$ \\
\hline $\begin{array}{c}\text { La inmigración } \\
\text { El cambio climático }\end{array}$ & $34,0 \%$ & $35,0 \%$ \\
\hline
\end{tabular}

Media de respuestas para el año $2018(a+b)$ a la cuestión QA5 (los dos problemas que el encuestado considera principales para la UE) ${ }^{6}$

Fuente: Elaboración propia a partir de Eurobarómetro 2018 
Tabla 6. Media de respuestas para el año 2019 a la cuestión QA5 (los dos problemas que el encuestado considera principales para la UE)

\begin{tabular}{c|c|c|c|c|c}
\hline $\begin{array}{c}\text { Dos principales } \\
\text { problemas de la UE }\end{array}$ & UE 28 & UE 28-UK & FR & SK & SE \\
\hline $\begin{array}{c}\text { La situación económica } \\
\text { en mi país }\end{array}$ & $18,0 \%$ & $17,0 \%$ & $17,0 \%$ & $12,5 \%$ & $14,0 \%$ \\
\hline $\begin{array}{c}\text { Precios } \\
\text { crecientes/inflación/coste } \\
\text { de la vida }\end{array}$ & $10,5 \%$ & $11,0 \%$ & $17,0 \%$ & $17,5 \%$ & $2,0 \%$ \\
\hline $\begin{array}{c}\text { Terrorismo } \\
\begin{array}{c}\text { La influencia de la UE } \\
\text { en el mundo }\end{array}\end{array}$ & $11,5 \%$ & $11,0 \%$ & $10,0 \%$ & $10,5 \%$ & $16,0 \%$ \\
\hline $\begin{array}{c}\text { Situación financiera de } \\
\text { mi hogar }\end{array}$ & $16,5 \%$ & $17,0 \%$ & $12,5 \%$ & $17,5 \%$ & $14,9 \%$ \\
\hline Inmigración & $34,0 \%$ & $35,0 \%$ & $29,0 \%$ & $41,0 \%$ & $36,0 \%$ \\
\hline Cambio climático & $23,0 \%$ & $23,0 \%$ & $22,5 \%$ & $14,5 \%$ & $48,0 \%$ \\
\hline
\end{tabular}

Media de respuestas para el año 2019 a la cuestión QA5 (los dos problemas que el encuestado considera principales para la UE)

Fuente: Elaboración propia a partir de Eurobarómetro 2019

En el caso de la agenda europea, al contrario que en la agenda de problemas "personales", existen menores divergencias, pues son pocos los problemas o países que logran destacarse significativamente de las opiniones del conjunto. De esta forma, despunta la preocupación por la posición de la Unión Europea en el mundo por parte Suecia (16\% contra 11\% general en 2019) y por los impuestos y el coste de la vida en Francia y Eslovaquia.

Estos resultados indican que, desde el punto de vista de los ciudadanos, existe un consenso aceptable en la agenda de principales problemáticas que afronta la UE. A nivel de agenda "personal" encontramos ciertas similitudes, pero con un grado mayor de divergencia entre los distintos Estados, con un grupo de países del Norte que tienden a destacar issues climáticos y educativos (cuestiones por lo general posmaterialistas en los términos de Inglehart ${ }^{2}$ ) y conviven con un Sur orientado especialmente a temáticas económicas y materiales (situación económica del país y paro) y un conjunto informe y variado de países preocupados de forma intermitente por la inmigración. Es importante destacar que la grieta entre la ordenación de los temas que centran los problemas personales y los que son propios de la Unión Europea demuestra, nuevamente, la distancia entre 
la realidad diaria de la ciudadanía y el ámbito europeo, impidiendo que realmente se atienda a unos debates que no son completamente relevantes para la posición particular de los receptores de insumos informativos.

Resumiendo lo observado hasta el momento, en la Unión Europea existe un claro potencial para que pueda darse una esfera pública supranacional, ya que los ciudadanos europeos están de acuerdo en qué discutir y en qué les preocupa, pero no llega a articularse el adecuado proceso comunicativo al respecto porque, incluso siendo las motivaciones deliberativas equivalentes, persiste la voluntad de tratamiento nacional de las mismas.

La Unión cuenta con la materia prima de la opinión pública (los temas, los issues, las preocupaciones). Su problema fundamental es que no logra construir canales propicios para que esta opinión se exteriorice. Algunos de los factores que hemos apuntado previamente podrían ayudar a arrojar luz sobre el asunto. La falta de un canal o una forma de articular el potencial al que nos hemos referido puede vincularse con la ausencia de un sistema de medios europeo, la falta de rentabilidad para los medios nacionales de tratar cuestiones europeas que con demasiada frecuencia aparecen alejadas de las demandas personales, así como verse propiciada por un diseño institucional desideologizado que prima al Estado frente al ciudadano como elemento constitutivo y que, por tanto, desanima la discusión pública y por un más que notable gap identitario y de cultura política, al menos, entre Norte y Sur. Este último apunte también podría denotarse como una brecha entre naciones plenamente instaladas en el posmaterialismo y otras todavía dependientes de las necesidades más materiales y económicas. Las dos velocidades de Europa dificultan una comunicación exitosa entre realidades que son persistentemente diferentes.

Con todos estos resultados, la europeización de los debates nacionales o el fortalecimiento de la opinión pública europea podrá comprobarse a medida que:

i. Se produzca una convergencia entre los problemas personales que preocupan a los europeos en su día a día y los problemas que afronta la Unión Europea, siendo, además, los segundos, problemas con solución plausible.

ii. Se origine, como consecuencia del anterior punto, un incremento progresivo del foco mediático en los debates europeos que, en todo caso, necesitarían visibilizar a las instituciones de la Unión como entidades decisionales, receptivas a la movilización pública y responsables en términos de rendición democrática de cuentas (accountability). La opinión pública ha de constituirse indiscutiblemente como una fuente de legitimidad activa para las decisiones de las instituciones europeas. Además, el aumento de la rentabilidad mediática de los temas europeos puede impulsar la constitución de grandes corporaciones que sumen medios de diversos países miembros que, sin renunciar a su carácter nacional, se unan para buscar lineamientos editoriales supranacionales, lo que también mejorará los niveles de cohesión argumental de las familias políticas europeas. iii. Se acerquen los valores políticos de los países y de los ciudadanos y, de este modo, no sólo las inquietudes se alineen, sino que también lo hagan las actitudes individuales sobre los retos colectivos. A la cuestión de los valores debe añadirse de forma determinante la de la cultura política. Estas reflexiones vienen a asentar lo que ya Habermas (2001: 17) postuló y es que para él "la generación de una opinión pública europea depende de los inputs vitales que introduzcan los actores de la sociedad civil" y, simultáneamente, "la extensión de una esfera pública a escala europea requiere que ésta se inscriba en una cultura política compartida por todos". 
iv.Los ciudadanos europeos sientan que tienen un peso específico en el funcionamiento político de la Unión, apartándose sus instituciones de las pautas despolitizantes que alejan el debate y la influencia sobre temas conflictivos de la ciudadanía.

v. Las instituciones europeas sean capaces de definir su propia agenda y transmitirla a los gobiernos nacionales y a los medios de comunicación de los países.

vi.La identificación con la Unión Europea se impulse a través de una revolución comunicativa institucional dispuesta a ejercer un liderazgo visible y proactivo en un nivel cercano, es decir, se trata de bajar a las instituciones europeas a las arenas informativas locales, regionales o nacionales.

Los hallazgos obtenidos a través del análisis del Eurobarómetro (periodo 2018-2019) en los indicadores relativos a la frecuencia de discusión sobre temas europeos, las preocupaciones personales y los problemas que afronta la Unión Europea, demuestran empíricamente las teorías sostenidas por Habermas, el filósofo más distinguido de la esfera pública. Es imposible lograr un espacio de deliberación común mientras que los ciudadanos de los distintos Estados miembros no compartan los mismos problemas sociales relevantes, pero, especialmente, ya que ese nivel parece que ha iniciado un proceso avanzado de igualación, lo que todavía falta por lograr es que todos los públicos al mismo tiempo hablen de los mismos temas, incluso con perspectivas culturales (lógicamente también lingüísticas) múltiples. La coordinación armoniosa de las temáticas de la deliberación requiere, primero, de un aumento significativo de la atención al ámbito europeo y, para ello, este espacio ha de tratar de transformar sus clásicos problemas percibidos hasta lograr que los mismos sean coincidentes con las preocupaciones reales de los europeos, aquellas que fomentarán un continuo diálogo democrático y que se deben trasladar en forma de intercambios políticos, parlamentarios e ideológicos (con cierto nivel de conflictividad) de amplio reconocimiento social. Sobre todo ello parece interesante recuperar el siguiente fragmento de Habermas:

La función de la infraestructura de las comunicaciones en una esfera pública democrática es convertir problemas sociales relevantes en temas de preocupación y permitir que el público en general se preocupe al mismo tiempo de los mismos temas, tomando una posición positiva o negativa ante las noticias y las opiniones. Con el tiempo, estas actitudes implícitas coagulan hasta constituir la opinión pública, incluso cuando la mayoría de los ciudadanos no envían mensajes públicos aparte de votar o no votar (2001: 17).

\section{Conclusiones}

En la reflexión general sobre los problemas que viene enfrentando el proceso europeo de integración resulta necesario destacar más enfáticamente el desarrollo de una esfera pública supranacional como condición facilitadora. La pregunta de investigación que ha centrado este estudio (“¿existe una opinión pública europea?”) ha venido siendo tratada clásicamente a través de un enfoque cualitativo, teórico o de análisis de contenido centrado en los medios de comunicación.

Superando la línea general de estudios en la materia, y partiendo de un diálogo teórico consistente, se aporta una propuesta de investigación empírica sobre la cuestión objeto de estudio a partir de los datos de encuesta del Eurobarómetro en los dos últimos años disponibles. Tomando como base dos dimensiones fundamentales del concepto operacionalizado (la integración de la discusión sobre temas europeos en la vida cotidiana y el nivel de acuerdo entre los temas de la agenda pública), se obtienen indicadores que permiten constatar su situación en la actualidad.

Los resultados apuntan a que los temas europeos todavía están por detrás en frecuencia de discusión, lo que demuestra, al mismo tiempo, tanto la conocida falta de interés en lo relacionado 
con este ámbito como la falta de información. Mientras que lo europeo siga presente de forma marginal en la conversación diaria será altamente improbable la constitución de una suerte de esfera pública más allá de los países. Sin embargo, se ha podido concluir que los problemas personales que afectan a los encuestados son ciertamente similares entre los Estados Miembros, lo que parece, sin duda, un buen precedente para una deliberación supranacional. Incluso con este aceptable nivel de acuerdo, el estudio evidencia una brecha entre realidades nacionales más cercanas al posmaterialismo (las del Norte) y otras (las del Sur) todavía preocupadas mayoritariamente por los issues clásicos de la industrialización.

Con el análisis de los problemas que, según la percepción de los encuestados, afronta la Unión Europea, puede afirmarse la existencia de un consenso aceptable en la agenda de principales problemáticas que afronta la Unión. Eso sí, persiste cierta lejanía entre la ordenación de los temas que centran los problemas personales y los que son propios de la Unión Europea. A medida que la convergencia entre los retos de Europa y los retos personales crezca, será más fácil poder constituir una opinión pública europea.

En el apartado cuarto se ha tenido ocasión de señalar algunas circunstancias que impulsarían la europeización de los debates nacionales, que es la forma más actual de concebir una esfera pública europea plausible, no desde el presupuesto de la homogeneidad, sino desde la visión plural que no renuncia a la base nacional. Todas estas circunstancias pueden resumirse en tres grandes grupos, las que tienen que ver con el sistema de medios, las relacionadas con la arquitectura institucional y las relativas a la identidad cultural y los valores políticos.

Alcanzar una esfera pública europea, que no paneuropea, a día de hoy, no es una utopía, pero, por el momento, parece difícil. En este empeño se debería insistir ya que, hasta que los europeos no hablen sobre los mismos temas y en el marco de un imaginario mayoritario compartido, alcanzar una acción común y una esfera de gobierno autónoma más allá del Estado nación resultará un objetivo lejano.

\section{Referencias}

ADAM, Silke (2015). European Public Sphere. In Gianpietro MAZZOLENI. (ed. jefe), The International Encyclopedia of Political Communication (pp. 370-378). John Wiley \& Sons, Inc.

AGUILERA DE PRAT, Cesáreo (2004). ¿Existe un demos europeo?: una propuesta normativa. Revista de Estudios Políticos (Nueva Época), 125, 157-179.

ANDUIZA, Eva.; Ismael CRESPO y Mónica MÉNDEZ (2009). Metodología de la Ciencia Política. Madrid: Centro de Investigaciones Sociológicas.

ARREGUI, Javier (2012). Problemas de legitimidad democrática, representación y rendimiento de cuentas en el proceso político de la Unión Europea. Cuadernos Europeos de Deusto, 46, 85-112.

BAISNÉE, Oliver (2007). The European Public Sphere Does Not Exist (At Least It's Worth Wondering...). European Journal of Communication, 22(4), 493-503. https://doi.org/10.1177/0267323107083065

BARÓN GOIRIENA, Alejandro (2009). El problema del déficit democrático y su resolución parlamentaria en el seno de la Unión Europea. Revista Española de Relaciones Internacionales, 1, 175-195.

BØLSTAD, Jørgen (2015). Dynamics of European integration: Public opinion in the core and periphery. European Union Politics, 16(1), 23-44. https://doi.org/10.1177/1465116514551303

BULLER, Jim y Andrew GAMBLE (2002). Conceptualizing Europeanization. Public Policy and Administration, 17 (2), 4-24. https://doi.org/10.1177/095207670201700202 
ERIKSEN, Erik Oddvar (2005). An Emerging European Public Sphere. European Journal of Social Theory, 8(3), 341-363. https://doi.org/10.1177/1368431005054798

FERNÁNDEZ OSTOS, María Teresa. (ed.lit) (2013). Sobre la posibilidad de una Esfera Pública Europea. Conversación entre Juan Torres y Sami Naïr. IC Revista Científica de Información y Comunicación, 10, 16-34.

GRIPSRUD, Jostein (2007). Television and the European Public Sphere. European Journal of Communication, 22 (4), 479-492. https://doi.org/10.1177/0267323107083064

HABERMAS, Jürgen (1989). The Public Sphere. In Steven Seidman (ed.), Jürgen Habermas on Society and Politics: A reader. Boston: Beacon Press.

HABERMAS, Jürgen (2001). ¿Por qué Europa necesita una constitución? New Left Review. 11, 5-25.

INNERARITY, Daniel (2017). La democracia en Europa. Barcelona: Galaxia Gutenberg.

KOOPMANS, Ruud (2007). Who inhabits the European public sphere? Winners and losers, supporters and opponents in Europeanised political debates. European Journal of Political Research, 46, 183-210. http s://doi.org/10.1111/j.1475-6765.2006.00691.x

MARSH, Michael. (1998). Testing the Second-Order Election Model after Four European. British Journal of Political Science, 28 (4), 591-607.

MORA, Alberto y MORENO, Cristina (2016). ¿Qué es la opinión pública? En Crespo, I.; Martínez, V.; Moreno, A.; Moreno, C. y Rabadán, R., Manual de herramientas para la investigación de la opinión pública (pp. 15-30). Valencia: Tirant Lo Blanch.

NITOIU, Cristian (2013). The European Public Sphere: Myth, Reality or Aspiration? Political Studies Review. 11(1), 26-38. https://doi.org/10.1111/j.1478-9302.2012.00287.x

OJALA, Markus (2013). ¿La crítica como medio para la democratización? La crisis del Euro y las perspectivas para la Esfera Pública Europea. IC Revista Científica de Información y Comunicación, 10, 73-98.

REIF, Karlheinz y Hermann SCHMITT (1980). Nine second order national elections - a conceptual framework for the análisis of European elections results. European Journal of Political Research, 3 (44), 3-44.

RODRÍGUEZ-AGUILERA, Cesáreo. (2015). El déficit democrático europeo. Madrid: Catarata.

SEDDONE, Antonella, Giuliano BOBBA y Franca RONCAROLO (2019). European versus Domestic Politics: Media Negativity during the 2019 European Elections Campaign in Italy. Italian Political Science, 4 (2), 74-92.

SIMÓN, Pablo. (2018). El príncipe moderno, democracia, política y poder. Barcelona: Penguin Random House.

TRENZ, Hans-Jörg (2004). Media Coverage on European Governance: Exploring the European Public Sphere in National Quality Newspapers. European Journal of Communication, 19 (3), 291-319.

ZUGASTI, Ricardo y Patricia LAFUENTE (2011). Los líderes políticos en las elecciones europeas de 2009. Miguel Hernández Communication Journal, 2, 141-153. Recuperado el 1 de julio de 2020 de: http://m hcj.es/2011/09/24/zugasti_lafuente/

Notas

1 Ver El País (23 de agosto de 1994) “El filósofo Jürgen Habermas dice que es importante que se cree una opinión pública común en Europa”. Recuperado de: https://elpais.com/diario/1994/08/23/ cultura/777592802_850215.html (06/07/2020).

2 Ver Inglehart, Ronald. (1990). Culture Shift in Advanced Industrial Society. New Jersey: Princeton University Press.

3 Los resultados están expresados como media entre los dos estudios que se realizan cada año ( $a+b / 2)$, de tal forma que el resultado anual no es un dato directo, sino elaborado a partir de dos componentes. Conviene 
puntualizar que la muestra $(\mathrm{N})$ de cada una de las encuestas se sitúa en el entorno de las 27.000 entrevistas distribuidas proporcionalmente según el peso poblacional de los países.

De nuevo, los resultados para cada año son la expresión de una media entre dos encuestas. No se seleccionan todos los países, sino aquellos cuyos datos aportan información relevante respecto de la tendencia general UE-28. Las abreviaturas usadas se corresponden con el código de países del Eurobarómetro, donde, por ejemplo, CY es Chipre, IE es Irlanda, EL es Grecia o RO es Rumanía. Para lectores no familiarizados con este sistema de códigos, se recomienda visitar la página del Eurobarómetro en alguno de sus informes: https:// www.europarl.europa.eu/at-your-service/es/be-heard/eurobarometer

5 En esta tabla solo se incluyen los principales problemas que para el encuestado enfrenta la Unión Europea como media del conjunto de los países que forman la muestra.

6 A diferencia de la anterior, las tablas 5 y 6 incorporan algunos países con resultados particulares que posteriormente se destacan en el análisis sobre la definición de la agenda de retos europeos que resulta particular en algunas casos como el de Francia o el de Eslovaquia. 